Recepción: 20 / 12 / 2018

Aceptación: 28 / 01 / 2019

(c)

Ciencias de la salud

Publicación: 20 / 03 / 2019

Artículo de revisión

\title{
Diagnóstico y tratamiento adecuado en el control de enfermedades infecciosas
}

\section{Diagnosis and adequate treatment in the control of infectious diseases}

\section{Diagnóstico e tratamento adequado no controle de doenças infecciosas}

Andrea C. Mariño-Jara ${ }^{\mathrm{I}}$ andybb.caro@gmail.com

Andrés P. Morales-Tipan II andres_pmt@hotmail.com

María E. Oña-Rivas III ma.eu.ona.riva@gmail.com

Adriano A. Alarcón-Romero IV dr.aalarcon17@gmail.com

Jenny E. Landazuri-Barre V jelb1388@hotmail.com

Zoila A. Moncayo-Parraga VI jemmito21@hotmail.com

Correspondencia: andybb.caro@gmail.com

\footnotetext{
I. Médico Residente; Hospital Provincial General; Latacunga, Ecuador.

II. Médico General en Funciones Hospitalarias Área Traumatología; Hospital IESS; Ibarra, Ecuador.

III. Médico Residente Área de Emergencia; Hospital IESS; Portoviejo, Ecuador.

IV. Médico Residente del Área de Emergencia; Hospital IESS; Portoviejo, Ecuador.

v. Médico Residente de Otorrinolaringología y Cirugía Estética Facial en Otorrinos Pichincha.

VI. Médico Cirujano; Resiente en el Área de Emergencia Hospitalaria IESS; Portoviejo, Ecuador.
} 


\title{
Resumen
}

Las enfermedades infecciosas son enfermedades causadas por microrganismos. El diagnóstico etiológico de una enfermedad infecciosa y sus correspondientes exámenes de laboratorio varían según el aparato y sistema afectado. La evaluación bacteriológica requiere diferentes pruebas de laboratorio para identificar los microorganismos responsables de la infección. Dependiendo de los síntomas, se solicitará muestras de sangre, orina, esputo, de algún tejido o fluido corporal. El marcado isotópico de leucocitos es una técnica valiosa para detectar infecciones. La resonancia magnética se usa para diagnosticar y seguir enfermedades como osteomielitis, fiebre de origen desconocido y prótesis vasculares infectadas. La tuberculosis se transmite a través del aire cuando personas con tuberculosis pulmonar o de las vías respiratorias tosen, hablan, cantan, escupen o estornudan. El diagnóstico de sida es clínico y de laboratorio. A la evidencia serológica repetida se suman signos, síntomas y diagnósticos característicos, que combinados permiten distinguir la infección por VIH. Los antibióticos son la forma más eficaz de combatir las infecciones causadas por bacterias, eliminan las bacterias o dificultan su desarrollo y reproducción. Como debilidad, no son eficientes contra virus, hongos y/o parásitos. Para la hepatitis A no hay ningún tratamiento específico para esta enfermedad. Los síntomas pueden remitir lentamente a lo largo de varias semanas o meses. Lo más importante consiste en evitar medicación innecesaria, no se debe administrar antieméticos ni paracetamol. El lavado de manos es la medida más simple y menos costosa para prevenir infecciones asociadas a cuidados de salud. En esta investigación se usó una metodología descriptiva, con un enfoque documental, es decir, revisar fuentes disponibles en la red, como google académico, con contenido oportuno y relevante desde el punto de vista científico.

Palabras claves: Microrganismos; Virus; Diagnóstico; Tratamiento; Cultivo; Antibióticos; Pruebas Bacterianas; Supervisión Médica.

\begin{abstract}
Infectious diseases are diseases caused by microorganisms. The etiological diagnosis of an infectious disease and its corresponding laboratory tests vary according to the affected system and system. Bacteriological evaluation requires different laboratory tests to identify the microorganisms responsible for the infection. Depending on the symptoms, samples of blood, urine, sputum, tissue or body fluid will be requested. . The isotopic labeling of leukocytes is a valuable technique to detect infections. Magnetic resonance imaging is used to diagnose and follow diseases such as
\end{abstract}


Andrea C. Mariño-Jara; Andrés P. Morales-Tipan; María E. Oña-Rivas; Adriano A. Alarcón-Romero; Jenny E. Landazuri-Barre; Zoila A. Moncayo-Parraga

osteomyelitis, fever of unknown origin, and infected vascular prostheses. Tuberculosis is spread through the air when people with pulmonary or airway tuberculosis cough, talk, sing, spit or sneeze. The diagnosis of AIDS is clinical and laboratory. To the repeated serological evidence are added signs, symptoms and characteristic diagnoses, which combined allow us to distinguish HIV infection. Antibiotics are the most effective way to fight infections caused by bacteria, eliminate bacteria or hinder their development and reproduction. As a weakness, they are not efficient against viruses, fungi and / or parasites. For hepatitis A there is no specific treatment for this disease. Symptoms may subside slowly over several weeks or months. The most important thing is to avoid unnecessary medication, you should not administer antiemetics or acetaminophen. Hand washing is the simplest and least expensive measure to prevent infections associated with health care. In this research, a descriptive methodology was used, with a documentary approach, that is, to review sources available on the web, such as academic google, with timely and relevant content from the scientific point of view.

Keys words: Micro-Organisms; Virus; Diagnosis; Treatment; Culture; Antibiotics; Bacterial Tests; Medical Supervision.

\section{Resumo.}

Doenças infecciosas são doenças causadas por microorganismos. O diagnóstico etiológico de uma doença infecciosa e seus correspondentes exames laboratoriais variam de acordo com o sistema e sistema afetado. A avaliação bacteriológica requer diferentes testes laboratoriais para identificar os microrganismos responsáveis pela infecção. Dependendo dos sintomas, amostras de sangue, urina, escarro, tecido ou fluido corporal serão solicitadas. . A marcação isotópica de leucócitos é uma técnica valiosa para detectar infecções. A ressonância magnética é utilizada para diagnosticar e acompanhar doenças como osteomielite, febre de origem desconhecida e próteses vasculares infectadas. A tuberculose é transmitida pelo ar quando pessoas com tuberculose pulmonar ou das vias aéreas tossem, falam, cantam, cospem ou espirram. O diagnóstico da AIDS é clínico e laboratorial. Às repetidas evidências sorológicas somam-se sinais, sintomas e diagnósticos característicos, que combinados nos permitem distinguir a infecção pelo HIV. Os antibióticos são a maneira mais eficaz de combater infecções causadas por bactérias, eliminar bactérias ou impedir seu desenvolvimento e reprodução. Como uma fraqueza, eles não são eficientes contra vírus, fungos e / ou parasitas. Para a hepatite A não há tratamento específico para esta doença. Os sintomas podem 
diminuir lentamente ao longo de várias semanas ou meses. O mais importante é evitar medicação desnecessária, você não deve administrar antieméticos ou paracetamol. A lavagem das mãos é a medida mais simples e menos onerosa para prevenir infecções associadas aos cuidados de saúde. Nesta pesquisa, utilizou-se uma metodologia descritiva, com abordagem documental, ou seja, revisar fontes disponíveis na web, como o google acadêmico, com conteúdo oportuno e relevante do ponto de vista científico.

Palavras chaves: Microorganismos; Vírus; Diagnóstico; Tratamento; Cultura; Antibióticos; Testes bacterianos; Supervisão Médica.

\section{Introducción.}

Estamos expuestos durante toda nuestra jornada diaria a virus, bacterias, hongos y demás microorganismos, incluso en nuestro hogar por más limpio que este. El reconocer esta realidad no implica que las personas deban vivir en condiciones de laboratorio, sino más consientes a los diversas medidas que contribuyan a mejorar su salud. El lavado de manos es la medida más simple y menos costosa para prevenir infecciones asociadas a cuidados de salud.

El diagnostico etiológico es fundamental para confirmar las enfermedades infecciosas, sin embargo, se puede lograr un diagnostico apropiado por evaluación clínica. Los exámenes de laboratorio dan respuesta más certera siempre que se disponga de tiempo y equipo necesario para realizarlos. Por ejemplo, una fiebre de más de $38^{\circ} \mathrm{c}$ por varios días, requiere de un cultivo o pruebas de sangre según lo considere pertinente el médico tratante en el diagnóstico.

Algunas enfermedades como la tuberculosis se transmite a través del aire cuando personas con tuberculosis pulmonar o de las vías respiratorias tosen, hablan, cantan, escupen o estornudan. . El chikungunya es un virus que se mantiene en tejidos de articulaciones y causa artralgias prolongadas, asociadas a una respuesta inmune persistente. Otras enfermedades como el zika y el dengue se 
Andrea C. Mariño-Jara; Andrés P. Morales-Tipan; María E. Oña-Rivas; Adriano A. Alarcón-Romero; Jenny E. Landazuri-Barre; Zoila A. Moncayo-Parraga

transmiten por picadura de un zancudo. El cólera se transmite por la ingesta de agua y alimentos contaminados.

Todos los hábitos saludables son costosos en término de tiempo y dinero pero más económico que un tratamiento, lo importante es que ante cualquier síntoma o padecimiento que sienta que afecte su salud, acuda a un profesional serio que cuenta con el conocimiento y buena intención de ayudar a su paciente a recobrar su salud. A lo largo del desarrollo del presente artículo, se explicará detalladamente información relevante.

\section{Metodología.}

Esta investigación está dirigida al estudio del tema Diagnóstico y tratamiento adecuado en el control de enfermedades infecciosas. Para realizarlo se usó una metodología descriptiva, con un enfoque documental, es decir, revisar fuentes disponibles en la red, como google académico, con contenido oportuno y relevante desde el punto de vista científico para dar respuesta a lo tratado en el presente artículo y que sirvan de inspiración para realizar otros proyectos. Las mismas pueden ser estudiadas al final, en la bibliografía.

\section{Resultados.}

El diagnostico etiológico es fundamental para confirmar las enfermedades infecciosas, sin embargo, se puede lograr un diagnostico apropiado por evaluación clínica. Los exámenes de laboratorio dan respuesta más certera siempre que se disponga de tiempo y equipo necesario para realizarlos. Las muestras de laboratorio más solicitadas en patologías infecciosas son: cultivo y análisis citoquímicos 
del líquido cefalorraquídeo, frotis del mucus fetal, cultivo de heces, esputo, liquido pleural, sangre que unidos al diagnóstico clínico son una herramienta poderosa para el diagnóstico microbiológico. El diagnóstico etiológico de una enfermedad infecciosa y sus correspondientes exámenes de laboratorio varían según el aparato y sistema afectado.

\section{Diagnóstico clínico de las enfermedades infecciosas:}

Para el diagnóstico de las enfermedades infecciosas es necesario evaluar exhaustivamente pues de ello depende el tratamiento y curación del paciente. Los aspectos a evaluar son:

Manifestaciones clínicas: el médico tratante dependiendo de los síntomas, resultados de la evaluación física y factores de riesgo de la persona (sexo, edad, empleo o forma de trabajo, viajes, mascotas, vida sexual, salubridad del agua y alimentos consumidos, etc.). Confirmado el diagnóstico, el paciente tiene una infección, se requiere precisar el microorganismo responsable de la infección.

Evaluación bacteriológica: son necesarias diferentes pruebas de laboratorio para identificar los microorganismos responsables de la infección. Dependiendo de los síntomas, se solicitará muestras de sangre, orina, esputo, de algún tejido o fluido corporal. Esas muestras se pueden (Hazen, 2014):

1. Teñir y examinar al microscopio.

2. Cultivar, someter al microorganismo a condiciones que estimulen su crecimiento.

3. Comprobar si existen anticuerpos producidos por el sistema inmunológico del individuo en respuesta al microorganismo. 
Andrea C. Mariño-Jara; Andrés P. Morales-Tipan; María E. Oña-Rivas; Adriano A. Alarcón-Romero; Jenny E. Landazuri-Barre; Zoila A. Moncayo-Parraga

4. Detectar la presencia de antígenos de un microorganismo: no todos los microorganismos pueden cultivarse, en este caso, el diagnóstico se obtiene mediante pruebas inmunológicas cuyo objetivo es detectar uno de los siguientes elementos:

- Anticuerpos: producidos por el sistema inmunológico del paciente en respuesta a los microorganismos.

- Antígenos: moléculas del microorganismo que pueden desencadenar una respuesta inmunitaria en el cuerpo.

5. Detectar material genético del microorganismo: son pruebas basadas en ácidos nucleicos, no aplica para este tipo de microorganismo cualquiera de las otras pruebas. El material genético se descompone en ácido desoxirribonucleico (ADN) o ácido ribonucleico (ARN). Cada prueba genética es especifica de un microorganismo concreto, es decir, una determinación genética para el virus de la hepatitis C detecta sólo ese virus y no otro.

Existen otras pruebas utilizadas para identificar microorganismos distintos a las explicadas anteriormente, se denominan pruebas de identificación no basadas en ácido nucleicos:

$\checkmark$ Sustancias que hacen crecer el microorganismo cuando se cultiva.

$\checkmark$ Enzimas: producidas por el microorganismo que infectan las células o las propagan dentro de los tejidos en forma acelerada.

$\checkmark$ Otras sustancias presentes en el microorganismo (proteínas y ácidos grasos) que permiten su identificación.

6. Evaluación radiológica y otros métodos: la imagenología como los métodos in vitro son instrumentos de medicina nuclear para diagnosticar enfermedades infecciosas. El marcado isotópico de leucocitos es una técnica valiosa para detectar infecciones. Se marca una 
muestra de glóbulos blancos con tecnecio $99 \mathrm{~m}$, un radioisótopo de uso médico el cual se reinyecta en el paciente.

La resonancia magnética se usa para diagnosticar y seguir enfermedades como osteomielitis, fiebre de origen desconocido y prótesis vasculares infectadas. Resulta muy acertada en fiebres de origen tumoral (enfermedad de Hodgkin), el linfoma no hogkiniano, el cáncer colorrectal y el sarcoma.

\section{Enfermedades infecciosas y sus diagnósticos}

Tuberculosis (TB): la tuberculosis es una infección bacteriana crónica causada principalmente por Mycobacterium tuberculosis y ocasionalmente por 2 Coinfección TB/VIH. Se caracteriza por producir una reacción de hipersensibilidad mediada por células y granulomas en los tejidos afectados. El bacilo de la tuberculosis, M, es un aerobio estricto que como todas las micobacterias se distingue por sus lípidos de superficie que lo hacen resistente a la decoloración por el ácido-alcohol de ciertas tinciones, razón por la que se lo conoce también como bacilo ácido alcohol resistente (PAHO, 2010).

La tuberculosis se transmite a través del aire cuando personas con tuberculosis pulmonar o de las vías respiratorias tosen, hablan, cantan, escupen o estornudan. Esto produce núcleos de microgotas menores a 5 micras que contienen bacilos tuberculosos y que son inhaladas por personas en contacto con el enfermo. La tuberculosis por $\mathrm{M}$.bovis se produce por ingestión de leche o productos lácteos no pasteurizados provenientes de ganado enfermo. La tuberculosis extrapulmonar, con excepción de la laríngea, no se considera transmisible.

El diagnóstico de la TB pulmonar en las personas con VIH se realiza de la misma forma que en las personas sin VIH y se basa en: 
Andrea C. Mariño-Jara; Andrés P. Morales-Tipan; María E. Oña-Rivas; Adriano A. Alarcón-Romero; Jenny E. Landazuri-Barre; Zoila A. Moncayo-Parraga

Manifestaciones clínicas: los síntomas más importantes en una persona con TB pueden limitarse a fiebre, tos reciente, pérdida de peso y diaforesis nocturna. La tos crónica y la hemoptisis son menos frecuentes porque hay menos cavitación, inflamación e irritación endobronquial. El examen físico en general no ayuda a distinguir la TB pulmonar de otras infecciones pulmonares y a menudo no hay signos auscultatorios. A diferencia de la tuberculosis pulmonar en una persona sin VIH en la cual la sintomatología puede ser muy florida, los síntomas más importantes en una persona con VIH pueden limitarse a fiebre, tos reciente, pérdida de peso y diaforesis nocturna. La tos crónica y la hemoptisis son menos frecuentes porque hay menos cavitación, inflamación e irritación endobronquial. La tos en la persona con VIH siempre debe ser investigada, independientemente de sus características o duración, recogiendo muestras de esputo para el diagnóstico bacteriológico de TB. El examen físico en general no ayuda a distinguir la TB pulmonar de otras infecciones pulmonares y a menudo no hay signos auscultatorios.

Diagnóstico bacteriológico:

Baciloscopia: es el examen microscópico directo de muestras de esputo buscando bacilos ácidos alcohol resistente (BAAR) mediante la tinción de Ziehl Neelsen. Es de bajo costo, de fácil ejecución y los resultados pueden estar disponibles en pocas horas.

$\checkmark$ Cultivo: el cultivo de esputo para tuberculosis es mucho más sensible que la baciloscopia en el diagnóstico de la TB pulmonar, pudiendo incrementar la confirmación diagnóstica en aproximadamente 15 a 20\% (PAHO, 2010). Sin embargo, tiene un costo mayor, su accesibilidad es menor dado que requiere mayor capacidad técnica y tecnológica y requiere más tiempo para dar resultados (de 2 a 6 semanas dependiendo del método).

$\checkmark$ Diagnóstico radiológico y otros métodos: la TB pulmonar con VIH no presenta ningún signo radiológico patognomónico y el diagnóstico de la enfermedad no se realiza solo con 
un estudio radiológico. La radiografía de tórax sospechosa obliga a tomar en cuenta la clínica y el nexo epidemiológico, pero sobre todo a realizar los estudios microbiológicos en todos los pacientes. En la inmunodeficiencia leve la radiografía de tórax no es diferente a la de un paciente sin VIH (cavitaciones, infiltrados en los vértices). En la inmunodeficiencia severa el aspecto de la radiografía de tórax es a menudo atípico, predominando la afección linfática y los signos de diseminación hematógena (infiltrado intersticial difuso o patrón miliar).

Síndrome de inmuno deficiencia adquirida (Sida): es una enfermedad infecciosa que causa deterioro funcional constante y progresivo de los linfocitos CD4 tanto desde el punto de vista cuantitativo como cualitativo, aunado al deterioro secundario de todo el sistema inmune celular. Los mecanismos patogénicos del deterioro de los linfocitos CD4 están relacionados directamente con un efecto citopático del VIH, pero existen otros mecanismos de destrucción indirecta implicados como causa del proceso de inmunosupresión (apoptosis secundaria a proteínas virales, alteraciones en la homeoestasis linfocitaria) ya que la destrucción linfocitaria por efecto citopático directo no explica todos los fenómenos de desregulación inmunitaria que se observan en el sida.

El VIH se transmite a través de sangre, semen y secreciones vaginales. También se encuentra la transmisión vertical (de madre a hijo). Una forma de transmisión importante, es a través del uso de drogas inyectables, específicamente por compartir jeringas contaminadas.

Diagnóstico de sida: el diagnóstico de sida es clínico y de laboratorio. A la evidencia serológica repetida se suman signos, síntomas y diagnósticos característicos, que combinados permiten distinguir la infección por VIH. Esta distinción tiene importancia clínica, ya que el diagnóstico de sida debe conducir al pronto establecimiento de tratamiento antirretroviral e importancia 
Andrea C. Mariño-Jara; Andrés P. Morales-Tipan; María E. Oña-Rivas; Adriano A. Alarcón-Romero; Jenny E. Landazuri-Barre; Zoila A. Moncayo-Parraga

epidemiológica, porque el registro de las personas con la infección asintomática por VIH da una información invalorable para determinar las tasas de transmisión recientes (Medina, 2012).

Zika: es una infección causada por un flavivirus transmitido por mosquitos del género Aedes, es asintomática en un $80 \%$ de los casos (Kantor, 2016). La enfermedad se manifiesta con exantema, conjuntivitis y fiebre no muy elevada, los síntomas duran entre 2 a 7 días. Se puede transmitir en humanos por vía parental, sexual y por trasfusión de sangre. Se diagnóstica por RT-PCR y por aislamiento del virus en muestra de sangre. El diagnostico serológico es inseguro por las reacciones cruzadas con otros flavivirus, dengue, fiebre amarilla.

Chikungunya: es un alfa virus, del que existen tres genotipos: africano, asiático y central. Lo trasmiten los mosquitos A. aegypti y A. albopictus, inicialmente su trasmisión era sólo enzoótica con brotes aislados en humanos. A partir del año 2000 se extendió a humanos en brotes epidémicos. El virus se mantiene en tejidos de articulaciones y causa artralgias prolongadas, asociadas a una respuesta inmune persistente. Los biomarcadores de inflamación IL-1 beta e IL-6 pueden predecir la gravedad de la enfermedad pero no hay tratamiento efectivo. Los métodos de diagnóstico son el cultivo, el RT-PCR, MAC-ELISA y PRNT.

Cólera: es una enfermedad diarreica aguda causada por la ingesta de alimentos o agua contaminada con el bacilo Vibrio cholerae. La aparición de los síntomas puede tardar de 12 horas a 5 días. Puede afectar a niños y adultos. La transmisión del cólera esta aunada al acceso insuficiente de agua potable e instalaciones de saneamiento. Las zonas de riesgo son zonas marginales o barriadas y campos de personas refugiadas o desplazadas, donde no existe el agua salubre y saneamiento. 
La clave para prevenir el cólera, controlarlo y reducir las defunciones, consiste en adoptar un criterio multidisciplinario basado en la vigilancia, el agua, el saneamiento y la higiene, la movilización social, el tratamiento y la vacunación oral.

\section{Tratamientos}

Los antibióticos son la forma más eficaz de combatir las infecciones causadas por bacterias, eliminan las bacterias o dificultan su desarrollo y reproducción. Como debilidad, no son eficientes contra virus, hongos y/o parásitos. El médico debe realizar un diagnóstico específico para determinar cuál antibiótico es el más apropiado, que combata el microorganismo. Si el uso correcto del antibiótico no logra eliminar la bacteria (evolucionan), se requerirá nuevas pruebas de laboratorio para identificarla. A continuación se presentan los tratamientos de acuerdo a las enfermedades analizadas anteriormente.

El cólera: se trata en forma fácil, se recomienda la administración de sales de rehidratación oral (SRO). El sobrecito estándar de SRO de la Organización Mundial de la Salud (OMS) y de UNICEF se disuelve en un litro de agua potable. Los pacientes adultos pueden requerir hasta seis litros de SRO para tratar una deshidratación moderada en el primer día. Los enfermos con deshidratación grave, necesitan la administración de líquidos endovenoso lo más pronto posible.

Un adulto de $70 \mathrm{~kg}$ necesitara al menos 7 litros endovenosos más SRO durante el tratamiento. Los pacientes también deben recibir antibióticos adecuados para frenar la duración de la diarrea, reducir el volumen de líquidos de rehidratación necesarios y reducir la magnitud y duración de la diarrea.

Tuberculosis: existe un código estándar para los regímenes de tratamiento antituberculoso. Cada medicamento tiene una abreviatura, un régimen tiene dos fases y cada una consiste en una 
Andrea C. Mariño-Jara; Andrés P. Morales-Tipan; María E. Oña-Rivas; Adriano A. Alarcón-Romero; Jenny E. Landazuri-Barre; Zoila A. Moncayo-Parraga

combinación de medicamentos. El número antes de una fase es la duración de esa fase en meses. Un subnumeral después de una letra es el número de dosis semanales de ese medicamento. Si no hay un subnumeral después de una letra eso significa que el tratamiento con ese medicamento es diario. La Organización Panamericana de la Salud/Organización Mundial de la Salud (OPS/OMS) y la Unión Internacional contra la Tuberculosis y Enfermedades Respiratorias, recomiendan regímenes estandarizados. Se muestra una tabla a continuación que refleja el tratamiento sugerido.

\section{Tabla $N^{\circ}$ 1: Medicamentos antituberculosos de Primera línea}

\begin{tabular}{|c|c|c|c|c|c|c|}
\hline \multirow{2}{*}{$\begin{array}{c}\text { Medicamento } \\
\text { de } 1^{\circ} \text { línea }\end{array}$} & \multirow{2}{*}{$\begin{array}{l}\text { Modo de } \\
\text { acción }\end{array}$} & \multirow{2}{*}{$\begin{array}{l}\text { Poten } \\
\text { cia }\end{array}$} & \multicolumn{4}{|c|}{ Dosis recomendada } \\
\hline & & & Diaria & $\begin{array}{l}\text { Máxim } \\
\text { a } \\
\text { mg }\end{array}$ & $\begin{array}{l}\text { Intermiten } \\
\text { te }\end{array}$ & $\begin{array}{l}\text { Máxima } \\
\text { mg }\end{array}$ \\
\hline Isoniacida $\mathbf{H}$ & bactericida & alta & $5(4-6)$ & 300 & $10(8-12)$ & 900 \\
\hline Rifampicina $\mathbf{R}$ & bactericida & alta & $10(8-10)$ & 600 & $10(8-12)$ & 600 \\
\hline Pirazinamina $\mathrm{Z}$ & bactericida & baja & $\begin{array}{c}25(20- \\
30)\end{array}$ & - & & - \\
\hline Etambutol E & bacteriostático & baja & $\begin{array}{c}15(15- \\
20)\end{array}$ & - & $30(25-35)$ & - \\
\hline $\begin{array}{c}\text { Estreptomicina } \\
\text { S }\end{array}$ & bactericida & baja & $\begin{array}{c}15(12- \\
18)\end{array}$ & 1000 & & - \\
\hline
\end{tabular}

Fuente: OMS 2010

En el régimen para pacientes nuevos la fase inicial o intensiva (dos meses) con la combinación de cuatro medicamentos elimina rápidamente los bacilos en división activa, contribuye a la 
disminución de la contagiosidad, evita la selección de cepas resistentes a medicamentos, especialmente a la isoniacida, y contribuye a la mejoría clínica del paciente. En la segunda fase o de continuación (cuatro meses) se utilizan dos medicamentos con el fin de eliminar a los bacilos en división intermitente. En los casos de tuberculosis extrapulmonar con riesgo serio de discapacidad y de difícil evaluación de respuesta al tratamiento (tuberculosis ósea o articular) o con riesgo serio de mortalidad (tuberculosis del sistema nervioso central).

La segunda fase se puede prolongar hasta finalizar de 9 a 12 meses de tratamiento respectivamente. En la primera fase del tratamiento antituberculoso se recomienda administración diaria de la medicación; en la segunda fase se recomienda en forma diaria o al menos tres veces por semana. Todas las ingestas de medicamentos deben ser directamente observadas por el personal de salud o una persona capacitada para tal fin. NO se recomienda la administración bisemanal de la medicación antituberculosa.

Meningitis: debe considerarse siempre como una urgencia médica, se debe ingresar al paciente en un centro de salud, el tratamiento antibiótico apropiado debe comenzar lo más pronto posible, después de la punción lumbar, siempre que se pueda practicar. El inicio del tratamiento antes de la punción, dificulta el crecimiento de la bacteria en el cultivo de LCR y la confirmación del diagnóstico. Algunos antibióticos sugeridos: penicilina, ampicilina, cloranfenicol y ceftriaxona.

Hepatitis A: no hay ningún tratamiento específico para esta enfermedad. Los síntomas pueden remitir lentamente a lo largo de varias semanas o meses. Lo más importante consiste en evitar medicación innecesaria, no se debe administrar antieméticos ni paracetamol. La hospitalización es innecesaria en ausencia de insuficiencia hepática aguda. El tratamiento tiene como objetivo logar el bienestar y equilibrio nutricional del paciente, incluida la rehidratación tras vómitos y diarreas. 
Andrea C. Mariño-Jara; Andrés P. Morales-Tipan; María E. Oña-Rivas; Adriano A. Alarcón-Romero; Jenny E. Landazuri-Barre; Zoila A. Moncayo-Parraga

Ebola: hasta el presente no hay ningún tratamiento eficaz para esta enfermedad, lo que se aplica posteriormente a su diagnóstico es tratamiento de apoyo: rehidratación con líquidos orales o intravenosos, sin embargo, se evalúan actualmente varias formas de hemoterapia, inmunoterapia y farmacoterapia.

\section{Conclusión.}

El presente artículo abordo el diagnóstico y tratamiento de algunas enfermedades infecciosas como la tuberculosis, el VIH, el Ebola, el cólera, el zika, entre otras, si bien es cierto que existen muchas otras más, no se consideraron por espacio en la investigación, nada obedece a un criterio de considerarlas poco importante para el investigador.

El diagnóstico etiológico de una enfermedad infecciosa y sus correspondientes exámenes de laboratorio varían según el aparato y sistema afectado. Es necesario evaluar exhaustivamente pues de ello depende el tratamiento y curación del paciente. Los aspectos a evaluar son:

1. El médico tratante dependiendo de los síntomas, resultados de la evaluación física y factores de riesgo de la persona confirmado el diagnóstico.

2. Puede requerir pruebas de laboratorio:

- Anticuerpos: producidos por el sistema inmunológico del paciente en respuesta a los microorganismos.

- Antígenos: moléculas del microorganismo que pueden desencadenar una respuesta inmunitaria en el cuerpo.

- Cultivos 
- Pruebas genéticas de microorganismos: son pruebas basadas en ácidos nucleicos, no aplica para este tipo de microorganismo cualquiera de las otras pruebas.

3. La imagenología como los métodos in vitro son instrumentos de medicina nuclear para diagnosticar enfermedades infecciosas. El marcado isotópico de leucocitos es una técnica valiosa para detectar infecciones.

Algunas enfermedades como la tuberculosis se transmite a través del aire cuando personas con tuberculosis pulmonar o de las vías respiratorias tosen, hablan, cantan, escupen o estornudan. Otras como el VIH se transmiten a través de sangre, semen y secreciones vaginales. También se encuentra la transmisión vertical (de madre a hijo) y uso de drogas inyectables, específicamente por compartir jeringas contaminadas. El zika, chikungunya y dengue por picadura de un mosquito.

Los antibióticos son la forma más eficaz de combatir las infecciones causadas por bacterias, eliminan las bacterias o dificultan su desarrollo y reproducción. Como debilidad, no son eficientes contra virus, hongos y/o parásitos. El médico debe realizar un diagnóstico específico para determinar cuál antibiótico es el más apropiado, que combata el microorganismo. Si el uso correcto del antibiótico no logra eliminar la bacteria (evolucionan), se requerirá nuevas pruebas de laboratorio para identificarla. No todas las enfermedades infecciosas tienen cura, la hepatitis A, el Ebola, el VIH no tienen cura pero si tratamientos que alivian el padecimiento, la mayor dificultad esta su costo, es el caso del VIH.

\section{Bibliografia.}

Hazen K. (2014). Diagnóstico de las enfermedades infecciosas-infecciones. Recuperado por http: www.msdmanuals.com

Kantor I. (2016). Dengue, zika y chikungunya. Recuperado por http: www.medicinabuenosaires.com 
Andrea C. Mariño-Jara; Andrés P. Morales-Tipan; María E. Oña-Rivas; Adriano A. Alarcón-Romero; Jenny E. Landazuri-Barre; Zoila A. Moncayo-Parraga

Lasso M. (2011). Diagnóstico y tratamiento de infecciones oportunistas en pacientes adulto con infección de VIH. Recuperado por http: www.scielo.conicyt.cl

Medina J. (2012). Pautas para el diagnóstico y tratamiento de las infecciones oportunistas en pacientes con VIH. Recuperado por http: www.infectologia.edu.uy

Organización Panamericana de la Salud (PAHO). (2010). Coinfección de Tuberculosis y VIH. Recuperado por http: www.paho.org

Rodríguez A. (2012). Principios básicos para el diagnóstico de las enfermedades infecciosas. Recuperado por http: www.telmed.org

Roses M. (2011). La resistencia a los antimicrobianos: un factor de riesgo para las enfermedades infecciosas. Recuperado por http: www.scielop.org

Úriz J. (2007). Tuberculosis en pacientes con sida. Recuperado por http: $\underline{w w w . s c i e l o . e s}$

Velazco E. (2014). Enfermedad por virus chikungunya en España. Boletín de Epidemiológia Semanal, volumen 22, número 16. Recuperado por http: www.revista.isciii.es 